\title{
An Efficient Algorithm for Zero-Forcing Coordinated Beamforming
}

\author{
Haksoo Kim, Heejung Yu, Youngchul Sung, and Yong H. Lee
}

\begin{abstract}
The problem of beam tracking for zero-forcing coordinated beamforming in multiple-cell time-varying multipleinput multiple-output channels is considered. Based on nullspace perturbation theory, an efficient update algorithm for block-diagonalization-based zero-forcing coordinated beam design is proposed and its performance is analyzed. In slowlyvarying channels, the proposed algorithm well tracks the zeroforcing beam solution and significantly reduces computational complexity by simply updating beamforming matrices instead of redesigning them with new channel state information at each time step.
\end{abstract}

Index Terms-Coordinated beamforming, coordinated multipoint, time-varying channels, perturbation theory.

\section{INTRODUCTION}

$\mathbf{I}$ $\mathrm{N}$ emerging wireless communication networks with small cells, interference is one of the main causes of performance degradation, and thus handling interference properly is critical for the network design. Among several interference-handling schemes, coordinated processing among basestations (BSs) with multiple antennas has been studied extensively as one of the key techniques for the 3GPP LTE-Advanced standard under the name of cooperative multipoint (CoMP) transmission [1]. For the downlink CoMP transmission, joint processing (JP) and coordinated beamforming (CB) schemes are mainly considered [2]. In JP, multiple BSs collaborate and transmit data to mobile-stations (MSs) in the collaborating cells with the assumption of full data and channel state information (CSI) sharing among the collaborating BSs. In the CB scheme, on the other hand, each BS designs its beamforming matrix to minimize the interference to other cell users based only on partial CSI, i.e. CSI from the served MS and interferenceexperiencing MSs, with no data sharing. The CB scheme is thus considered to be more practical in cases where inter-cell backhaul links have bandwidth and delay constraints.

In this letter, we consider the beam design problem for $\mathrm{CB}$ under the well-known zero-forcing (ZF) framework. Most previous works on $\mathrm{CB}$ have focused on the beam design problem in static channels [3], [4], [5]. However, real wireless channels are time-varying, and this time-varying nature of channels has not been considered extensively for CB. Here,

Manuscript received December 19, 2011. The associate editor coordinating the review of this letter and approving it for publication was N.-D. Dao.

H. Kim, Y. Sung (corresponding author), and Y. H. Lee are with the Dept. of Electrical Engineering, KAIST, Daejeon, 305-701, Korea (e-mail: \{hskim@stein,ysung@ee, yohlee@ee\}.kaist.ac.kr).

H. Yu is with ETRI, Daejeon, 305-700, Korea (e-mail: heejung@etri.re.kr).

This research was supported in part by the Basic Science Research Program through the National Research Foundation of Korea (NRF) funded by the Ministry of Education, Science and Technology (2010-0021269). This work was also supported in part by the IT R\&D program of MKE/KEIT. [2008-F004-02, "5G mobile communication systems based on beam division multiple access and relays with group cooperation"].

Digital Object Identifier 10.1109/LCOMM.2012.050412.112565 we consider the beam design for $\mathrm{ZF} \mathrm{CB}$ in time-varying multiuser multiple-input multiple-output (MIMO) channels, and propose an efficient beam tracking algorithm for ZF CB in time-varying MIMO channels, based on a similar technique to that developed for interference alignment in [7]; the previous results in [6], [7] only consider the eigenvector perturbation for square matrices and they are not directly applicable to the $\mathrm{ZF} \mathrm{CB}$, for which the null-space perturbation for fat matrices should be considered. Hence, in this letter we first derive a perturbation form for the null space of a fat matrix. Based on this derivation, we then propose a new beam tracking algorithm for $\mathrm{ZF} \mathrm{CB}$, and analyze the rate loss by the proposed tracking algorithm.

Notations: We make use of standard notational conventions. Vectors and matrices are written in boldface with matrices in capitals. All vectors are column vectors. For a matrix $\mathbf{A}$, $\mathbf{A}^{T}, \mathbf{A}^{H}$, and $\mathbf{A}^{\dagger}$ indicate the transpose, Hermitian transpose, and pseudo inverse of $\mathbf{A}$, respectively. I stands for the identity matrix. The notation $\mathrm{x} \sim \mathcal{C} \mathcal{N}(\boldsymbol{\mu}, \boldsymbol{\Sigma})$ means that $\mathrm{x}$ is Gaussiandistributed with a mean vector $\boldsymbol{\mu}$ and a covariance matrix $\boldsymbol{\Sigma}$. $\mathbb{E}$ denotes the expectation operator. $\mathbb{C}^{m \times n}$ is a set of $m \times n$ complex matrices.

\section{SySTEM MODEL}

We consider a multi-cell MIMO downlink network with $K$ BSs and $K$ MSs, where BS $k$ with $N_{k}$ antennas is paired with MS $k$ with $M_{k}$ antennas and each BS interferes with all undesired MSs. The received signal at MS $k$ at time $n$ is then given by

$$
\mathbf{y}_{k}[n]=\mathbf{H}_{k k}[n] \mathbf{V}_{k}[n] \mathbf{s}_{k}[n]+\sum_{l=1, l \neq k}^{K} \mathbf{H}_{k l}[n] \mathbf{V}_{l}[n] \mathbf{s}_{l}[n]+\mathbf{n}_{k}[n]
$$

where $\mathbf{H}_{k l}[n]$ denotes the $M_{k} \times N_{l}$ MIMO channel from BS $l$ to $\mathrm{MS} k ; \mathbf{V}_{l}[n]$ and $\mathbf{s}_{l}[n]$ are the $N_{l} \times d_{l}$ transmit beamforming matrix and the $d_{l} \times 1$ transmit data vector at BS $l$, respectively; $d_{l}$ denotes the number of data streams for the $l$-th BS-MS pair; and $\mathbf{n}_{k}(n)\left(\sim \mathcal{C N}\left(0, \sigma^{2} \mathbf{I}\right)\right)$ is the $M_{k} \times 1$ additive white Gaussian noise vector. For simplicity, we assume that $N_{l}=N=K M, M_{l}=M$ and $d_{l}=M$ for all $l$.

The ZF-based CB technique is an extension of the ZF beamforming method in the single-cell MIMO broadcast channel [8] to the multi-cell MIMO downlink. In the ZF-based CB, the interference to the undesired receivers is eliminated by designing $\mathbf{V}_{k}$ at $\mathrm{BS} k$ such that

$$
\tilde{\mathbf{H}}_{k}[n] \mathbf{V}_{k}[n]=\mathbf{0}, \quad k=1, \cdots, K,
$$


where $\tilde{\mathbf{H}}_{k}[n] \in \mathbb{C}^{(K-1) M \times N}$ is the composite interference channel matrix at $\mathrm{BS} k$, defined as

$$
\tilde{\mathbf{H}}_{k}[n] \triangleq\left[\mathbf{H}_{1 k}^{T}[n], \cdots, \mathbf{H}_{(k-1) k}^{T}[n], \mathbf{H}_{(k+1) k}^{T}[n], \cdots, \mathbf{H}_{K k}^{T}[n]\right]^{T} .
$$

Thus, a necessary and sufficient condition for ZF CB is that the column space of $\mathbf{V}_{k}[n]$ lies in the null space of $\tilde{\mathbf{H}}_{k}[n]$, and such a beamforming matrix can be obtained by subspace decomposition of $\tilde{\mathbf{H}}_{k}[n]$. ${ }^{1}$ The received signal at MS $k$ is free from interference from undesired transmitters when all the transmitters design their beamforming matrices to satisfy (2).

\section{BEAM TRACKING FOR ZF CB}

In time-varying channels, the beamforming matrix $\mathbf{V}_{k}[n]$ should be designed at each time step to accommodate the channel variation. One could design the beamforming matrix by performing subspace decomposition of $\tilde{\mathbf{H}}_{k}[n]$ at each time step. However, this method is computationally expensive, and the similarity between the two null spaces of two consecutive time steps reduces the computational complexity. To this end, we use and extend the null space perturbation result in [6], [7] to represent the perturbed null space of a fat matrix as an additive update form based on the channel perturbation.

Theorem 1: Let $\mathbf{V}_{k}[m]$ be a solution of equation (2) at time $m$ with channel $\left\{\mathbf{H}_{l k}[m]\right\}$; i.e. the columns of $\mathbf{V}_{k}[m]$ form a basis of the null space of $\tilde{\mathbf{H}}_{k}[m]$. A solution to (2) at time $n$ $(n>m)$ with channel $\left\{\mathbf{H}_{l k}[n]\right\}$ satisfying a quasi-orthogonal condition $\mathbf{V}_{k}[m]^{H} \mathbf{V}_{k}[n]=\mathbf{I}$ is given by

$$
\mathbf{V}_{k}[n]=\mathbf{V}_{k}[m]-\tilde{\mathbf{H}}_{k}[m]^{\dagger} \Delta \tilde{\mathbf{H}}_{k}[n, m] \mathbf{V}_{k}[m]+O\left(\left\|\Delta \tilde{\mathbf{H}}_{k}[n, m]\right\|_{2}^{2}\right),
$$

where $\Delta \tilde{\mathbf{H}}_{k}[n, m] \triangleq \tilde{\mathbf{H}}_{k}[n]-\tilde{\mathbf{H}}_{k}[m]$.

Theorem 1 is applicable to any case of $N_{\text {row }} \leq N_{\text {col }}$, where $N_{\text {row }}$ and $N_{\text {col }}$ are the numbers of rows and columns of $\tilde{\mathbf{H}}_{k}[m]$, respectively, and it is a generalized version of the result for $N_{\text {row }}=N_{\text {col }}$ in [6]. The second term in the right-hand side (RHS) in (4) represents the first-order estimate for the null space update due to the channel variation $\Delta \tilde{\mathbf{H}}_{k}[n, m]$, and the residual error increases with the order of $O\left(\left\|\Delta \tilde{\mathbf{H}}_{k}[n, m]\right\|_{2}^{2}\right)$. Theorem 1 can be proved using a similar technique to that used in [6], and here we just provide a sketch of the proof. To prove the theorem, we first obtain a fundamental equation given by substituting $\tilde{\mathbf{H}}_{k}[n]=\tilde{\mathbf{H}}_{k}[m]+\epsilon \overline{\Delta \tilde{\mathbf{H}}_{k}[n, m]}$ and $\mathbf{V}_{k}[n]=\mathbf{V}_{k}[m]+\epsilon \mathbf{V}_{k}^{(1)}[n, m]+\epsilon^{2} \mathbf{V}_{k}^{(2)}[n, m]+\cdots$ into $\tilde{\mathbf{H}}_{k}[n] \mathbf{V}_{k}[n]=\mathbf{0}$. (Here, $\epsilon=\left\|\Delta \tilde{\mathbf{H}}_{k}[n, m]\right\|_{2}, \overline{\Delta \tilde{\mathbf{H}}_{k}[n, m]}=$ $\frac{1}{\epsilon} \Delta \tilde{\mathbf{H}}_{k}[n, m]$ and $\|\cdot\|_{2}$ denotes the matrix 2-norm.) $\mathrm{A}$ general solution to the fundamental equation is then found iteratively and the coefficient matrices are determined by the normalization equation $\mathbf{V}_{k}[m]^{H} \mathbf{V}_{k}[n]=\mathbf{I}$. In the step for obtaining the general solution, the following fact is used. The necessary and sufficient condition for a system $\mathbf{B y}=\mathbf{c}$

\footnotetext{
${ }^{1}$ If the dimension of the null space of $\tilde{\mathbf{H}}_{k}$ is one, $\mathbf{v}_{k}[n]$ is the unique solution to (2) with unit norm. Otherwise, there are multiple solutions to (2) in the form of $\mathbf{V}_{k}[n] \mathbf{T}_{k}[n]$, where $\mathbf{T}_{k}[n]$ a linear transform matrix with size (nullity of $\tilde{\mathbf{H}}_{k}[n]$ ) $\times d$. To improve the rate performance under the ZF constraint, $\mathbf{T}_{k}[n]$ should be design as the matrix composed of the right singular vectors of the effective channel $\mathbf{H}_{k k}[n] \mathbf{V}_{k}[n]$, and the transmit power should be distributed by the water-filling algorithm. In this paper, we only consider the computation of $\mathbf{V}_{k}$, which is necessary anyway even for this optimal ZF processing.
}

of linear equations to be feasible is for any $\mathbf{x}, \mathbf{x}^{H} \mathbf{c}=0$ whenever $\mathbf{x}^{H} \mathbf{B}=\mathbf{0}$. Moreover, the solution for the feasible linear equation is $\mathbf{y}=\mathbf{B}^{\dagger} \mathbf{c}+\mathbf{d}$ with $\mathbf{B d}=\mathbf{0}$. Theorem 1 gives a simple and efficient update formula for $\mathbf{V}_{k}[n]$ at time $n=m+1, m+2, \cdots$ based on $\mathbf{V}_{k}[m]$ :

$$
\hat{\mathbf{V}}_{k}[n]=\mathbf{V}_{k}[m]-\tilde{\mathbf{H}}_{k}[m]^{\dagger} \Delta \tilde{\mathbf{H}}_{k}[n, m] \mathbf{V}_{k}[m] .
$$

Once $\mathbf{V}_{k}[m]$ is obtained by subspace decomposition at time $m$, the beam solution at time $n(>m)$ can be obtained simply by (5). In this case, the error between the exact and updated beams increases with $O\left(\left\|\Delta \tilde{\mathbf{H}}_{k}[n, m]\right\|_{2}^{2}\right)$. However, this error can be reset by performing a subspace decomposition to obtain an exact beam solution again after a certain number of updates, say $L$ time steps, as in [7]. Here, the period $L$ of subspace decomposition can be determined judiciously based on the channel fading rate. Note that the proposed algorithm requires only matrix multiplication and addition during the updating phase. Thus, the complexity reduction by the proposed algorithm is significant compared with the full subspace decomposition method.

\section{Performance Analysis}

We here examine the performance of the proposed algorithm. First, let us examine the computational complexity of the proposed algorithm with the number of flops per time step as the complexity measure. As a reference, we consider the non-recursive method, which computes the exact null space of $\tilde{\mathbf{H}}_{k}[n]$ by singular value decomposition (SVD) at every time step. During the updating phase, each BS requires $4 K(K-1) M^{3}+K M^{2}$ flops to obtain the CB matrix for the proposed algorithm, whereas $\left(21 K^{2}-16 K+4\right) K M^{3}$ is required for the non-recursive SVD-based method [9] under the assumption that SVD is done by the Golub-Reinsch algorithm [9]. Since the number of BS is $K$, the overall system complexity per time step is $K$ times the number of flops per BS. Hence, the order of computational complexity for the nonrecursive SVD based scheme is $O\left(K^{4}\right)$ flops, whereas only $O\left(K^{3}\right)$ flops are required for the proposed algorithm at each symbol time. Fig. 1 shows the computational complexity of the proposed algorithm and non-recursive method with different $K$ when $M=1$. The reduction yielded by the proposed method is significant for large $K$, and becomes even larger when we consider the updating interval $L$.

Now, let us consider the sum rate loss incurred by using the proposed algorithm instead of obtaining the exact null space of $\tilde{\mathbf{H}}_{k}[n]$ by subspace decomposition. For simplicity of analysis, we consider the case of a single receive antenna, i.e., $M=1$. Since the beam vector obtained by the proposed algorithm is not exact during the updating phase, the beam vector error in this phase can cause a loss in the sum rate. To assess this rate loss in the time-varying channel, we adopt the widely-used first-order Gauss-Markov channel model [11], given by

$$
h_{k l}^{j}[n]=\alpha_{k l} h_{k l}^{j}[n-1]+\sqrt{1-\alpha_{k l}^{2}} u_{k l}^{j}[n], \quad n \geq 1,
$$

for the channel coefficient between the $j$-th antenna of BS $l$ and MS $k$, where $h_{k l}^{j}[0] \sim \mathcal{C N}(0,1), u_{k l}^{j}[n] \sim \mathcal{C N}(0,1)$, and $\alpha_{k l}(\in(0,1])$ is the fading correlation coefficient, which depends on the Doppler spread and the symbol duration [11]. 


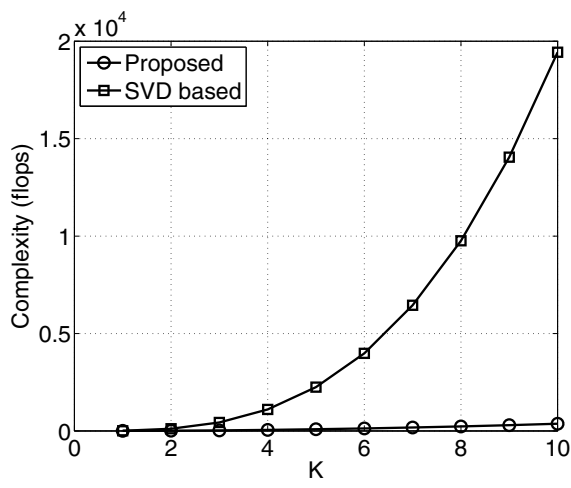

Fig. 1. Computational complexity versus $K$ when $M=1$.

We assume that the channel process for each MIMO channel element evolves independently and the fading correlation coefficients are identical for all $k, l$ as $\alpha$, i.e., $\alpha_{k l}=\alpha$ $\forall k, l$. Since the beam vector error increases with the channel difference, the expected sum rate loss is a function of the mobile speed $v_{m}$ and the time difference $p$ for given $K$ and $M$. The following theorem explains the impact of $v_{m}$ and $p$ on the expected sum rate.

Theorem 2: Let $v_{m}$ be the mobile speed for all users and $n=m+p, p \geq 1$. Under the assumption of the first-order Gauss-Markov channel model, the expected sum rate loss is given by

$$
\Delta R\left(p, v_{m}\right) \leq O\left(\log \left(1+c p^{2} v_{m}^{4}\right)\right),
$$

for some constant $c$, for given $K$ and $M$.

Proof: The expected sum rate loss at time $n$ is given by

$$
\begin{aligned}
& \Delta R\left(p, v_{m}\right) \\
& =\mathbb{E} \sum_{k=1}^{K} \log \left(1+\frac{\left|\mathbf{h}_{k k}^{H}[n] \mathbf{v}_{k}[n]\right|^{2}}{\sigma^{2}}\right)- \\
& \mathbb{E} \sum_{k=1}^{K} \log \left(1+\frac{\left|\mathbf{h}_{k k}^{H}[n] \hat{\mathbf{v}}_{k}[n]\right|^{2}}{\sum_{l=1, l \neq k}^{K}\left|\mathbf{h}_{k l}^{H}[n] \hat{\mathbf{v}}_{l}[n]\right|^{2}+\sigma^{2}}\right) \\
& \leq \mathbb{E} \sum_{k=1}^{K} \log \left(1+\frac{\left|\mathbf{h}_{k k}^{H}[n] \mathbf{v}_{k}[n]\right|^{2}}{\sigma^{2}}\right)-\mathbb{E} \sum_{k=1}^{K} \log \left(1+\frac{\left|\mathbf{h}_{k k}^{H}[n] \hat{\mathbf{v}}_{k}[n]\right|^{2}}{\sigma^{2}}\right) \\
& \quad+\mathbb{E} \sum_{k=1}^{K} \log \left(1+\frac{\sum_{l=1, l \neq k}^{K}\left|\mathbf{h}_{k l}^{H}[n] \hat{\mathbf{v}}_{l}[n]\right|^{2}}{\sigma^{2}}\right) \\
& =\mathbb{E} \sum_{k=1}^{K} \log \left(1+\frac{\sum_{l=1, l \neq k}^{K}\left|\mathbf{h}_{k l}^{H}[n] \tilde{\mathbf{v}}_{l}[n]\right|^{2}}{\sigma^{2}}\right) \\
& \leq \sum_{k=1}^{K} \log \left(1+\frac{\sum_{l=1, l \neq k}^{K} \mathbb{E}\left[\left\|\mathbf{h}_{k l}[n]\right\|^{2}\left\|\tilde{\mathbf{v}}_{l}[n]\right\|^{2}\right]}{\sigma^{2}}\right),
\end{aligned}
$$

where $\tilde{\mathbf{v}}_{k}[n]=\mathbf{v}_{k}[n]-\hat{\mathbf{v}}_{k}[n]$ denotes the error between the exact beam vector $\mathbf{v}_{k}[n]$ by SVD of $\tilde{\mathbf{H}}_{k}[n]$ and the updating solution $\hat{\mathbf{v}}_{k}[n]$ by (5). (9) is obtained by decomposing the numerator and denominator of the second term in the right hand side (RHS) of (8) and by using the fact $\left|\mathbf{h}_{k l}^{H}[n] \hat{\mathbf{v}}_{l}[n]\right|^{2} \geq 0$; (10) is obtained by the fact that the first two terms in the RHS of (9) cancel out ${ }^{2}$ and by substituting $\hat{\mathbf{v}}_{l}[n]=\mathbf{v}_{l}[n]-\tilde{\mathbf{v}}_{l}[n]$ (with the exact beam vector satisfying $\left.\mathbf{h}_{k l}^{H}[n] \mathbf{v}_{l}[n]=0\right)$ in the third term in the RHS of (9); (11) is

\footnotetext{
${ }^{2} \mathbf{v}_{k}[n]$ and $\hat{\mathbf{v}}_{k}[n]$ are isotropically distributed unit vectors and independent of $\mathbf{h}_{k k}[n]$ since they are designed based on $\left\{\mathbf{h}_{l k}[n], l \neq k\right\}$. Thus, the two terms cancel out [10].
}

obtained by sub-multiplicativity of norm (i.e. $\left|\mathbf{h}_{k l}^{H}[n] \tilde{\mathbf{v}}_{l}[n]\right|^{2} \leq$ $\left.\left\|\mathbf{h}_{k l}[n]\right\|^{2}\left\|\tilde{\mathbf{v}}_{l}[n]\right\|^{2}\right)$ and by Jensen's inequality.

Since the error of $\mathbf{v}_{l}[n]$ increases with $O\left(\left\|\Delta \tilde{\mathbf{H}}_{l}[n, m]\right\|_{2}^{2}\right)$, there exists $c^{\prime}$ such that ${ }^{3}$

$$
\left\|\tilde{\mathbf{v}}_{l}[n]\right\| \leq c^{\prime}\left\|\Delta \tilde{\mathbf{H}}_{l}[n, m]\right\|_{2}^{2} .
$$

Hence, $\mathbb{E}\left[\left\|\mathbf{h}_{k l}[n]\right\|^{2}\left\|\tilde{\mathbf{v}}_{l}[n]\right\|^{2}\right]$ is given by

$\mathbb{E}\left[\left\|\mathbf{h}_{k l}[n]\right\|^{2}\left\|\tilde{\mathbf{v}}_{l}[n]\right\|^{2}\right] \leq c^{\prime 2} \sqrt{\mathbb{E}\left\|\mathbf{h}_{k l}[n]\right\| 4 \mathbb{E}\left\|\Delta \tilde{\mathbf{H}}_{l}[n, m]\right\|_{F}^{8}}$.

where $\|\cdot\|_{F}$ denotes the Frobenius norm. In (13), we use the fact that $\|\mathbf{A}\|_{2} \leq\|\mathbf{A}\|_{F}$ for any matrix $\mathbf{A}$ and the Cauchy Schwartz inequality. Since $n=m+p$, from (6) $h_{k l}^{j}[n]$ and $\Delta h_{k l}^{j}[n] \triangleq h_{k l}^{j}[n]-h_{k l}^{j}[m]$ can be written as

$$
\begin{aligned}
h_{k l}^{j}[n] & =\alpha^{p} h_{k l}^{j}[m]+\sqrt{1-\alpha^{2 p}} \tilde{u}_{k l}^{j}[n] \text { and } \\
\Delta h_{k l}^{j}[n] & =\left(\alpha^{p}-1\right) h_{k l}^{j}[m]+\sqrt{1-\alpha^{2 p}} \tilde{u}_{k l}^{j}[n],
\end{aligned}
$$

respectively, where $\tilde{u}_{k l}^{j}[n] \sim \mathcal{C N}(0,1)$. Then, $h_{k l}^{j}[n]$ and $\Delta h_{k l}^{j}[n]$ are zero-mean complex Gaussian random variables with variance 1 and $2\left(1-\alpha^{p}\right)$, respectively. Hence,

$$
2\left\|\mathbf{h}_{k l}[n]\right\|^{2}=\sum_{j=1}^{K}\left|\sqrt{2} h_{k l}^{j}[n]\right|^{2} \sim \chi_{2 K}^{2}
$$

and

$$
\begin{aligned}
\frac{1}{1-\alpha^{p}}\left\|\Delta \tilde{\mathbf{H}}_{l}[n, m]\right\|_{F}^{2} & =\frac{1}{1-\alpha^{p}} \sum_{k=1, k \neq l}^{K} \sum_{j=1}^{K}\left|\Delta h_{k l}^{j}[n]\right|^{2} \\
& \sim \chi_{2 K(K-1)}^{2},
\end{aligned}
$$

where $\chi_{r}^{2}$ denotes the chi-square distribution with $r$ degrees of freedom. Since the $q$-th moment of the chi-square random variable $Z$ with $r$ degrees of freedom is $\mathbb{E}\left(Z^{q}\right)=2^{q} \frac{\Gamma(q+r / 2)}{\Gamma(r / 2)}$, (13) can be rewritten as

$$
\mathbb{E}\left[\left\|\mathbf{h}_{k l}[n]\right\|^{2}\left\|\tilde{\mathbf{v}}_{l}[n]\right\|^{2}\right] \leq c^{\prime \prime}\left(1-\alpha^{p}\right)^{2}
$$

where $c^{\prime \prime}=2^{2} c^{\prime 2} \sqrt{\frac{\Gamma(2+K)}{\Gamma(K)} \frac{\Gamma(4+K(K-1))}{\Gamma(K(K-1))}}$ and $\Gamma(\cdot)$ denotes the gamma function. In slowly-fading channels, i.e. $f_{d} T_{s} \ll 1$, the fading correlation coefficient $\alpha$ can be approximated as [12]

$$
J_{0}\left(2 \pi f_{d} T_{s}\right)=\sum_{r=0}^{\infty} \frac{(-1)^{r}}{2^{2 r}(r !)^{2}}\left(2 \pi f_{d} T_{s}\right)^{2 r} \approx 1-\left(\pi f_{d} T_{s}\right)^{2}
$$

where $f_{d}$ and $T_{s}$ denote the maximum doppler frequency and the symbol duration, respectively. Thus, we have $1-\alpha^{p} \approx$ $1-\left(1-\left(\pi f_{d} T_{s}\right)^{2}\right)^{p} \approx p\left(\pi f_{d} T_{s}\right)^{2}=\left(\frac{\pi T_{s}}{\lambda}\right)^{2} p v_{m}^{2}$, where $\lambda$ is the wave length. Hence, (11) is finally given by

$$
\begin{aligned}
\Delta R\left(p, v_{m}\right) & \leq K \log \left(1+\frac{(K-1) \pi^{4} T_{s}^{4} c^{\prime \prime}}{\sigma^{2} \lambda^{4}} p^{2} v_{m}^{4}\right) \\
& =O\left(\log \left(1+c p^{2} v_{m}^{4}\right)\right)
\end{aligned}
$$

where $c=\frac{(K-1) \pi^{4} T_{s}^{4} c^{\prime \prime}}{\sigma^{2} \lambda^{4}}$. Hence, we obtain (7) Theorem 2 explains the behavior of the expected sum rate loss caused by the proposed tracking algorithm in slowly-fading channels as a function of $p$ and $v_{m}$. The upper bound of the expected sum rate loss is a monotonically increasing function of $p^{2}$ and $v_{m}^{4}$. In the case of $p=0$ and $v_{m}=0$, there is no sum rate loss, as expected.

\footnotetext{
${ }^{3} \mathrm{~A}$ detailed information about $c^{\prime}$ can be found in [7].
} 


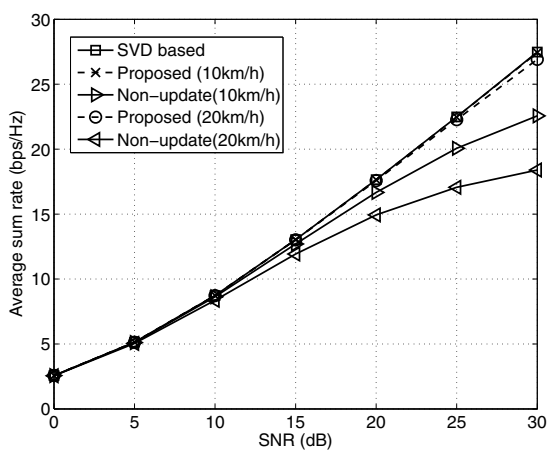

Fig. 2. Average sum rate w.r.t. SNR when $K=3, M=1$ and $L=100$.

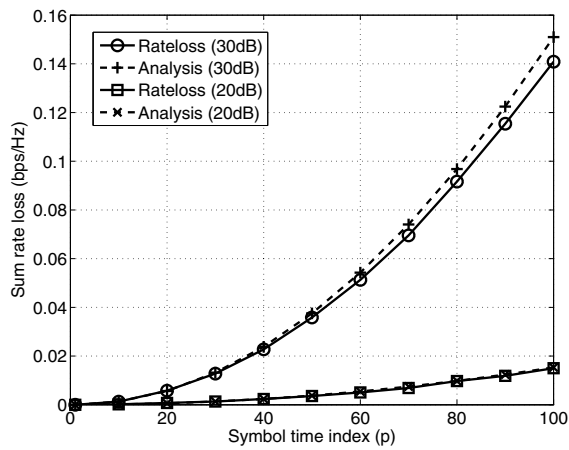

Fig. 3. Sum rate loss w.r.t. $p$ when $K=3, M=1$ and $v_{m}=10 \mathrm{~km} / \mathrm{h}$.

\section{Numerical Results}

In this section, we provide some numerical results to evaluate the performance of the proposed algorithm. We assume that the carrier frequency is $2.0 \mathrm{GHz}$ and the symbol duration is $66.7 \mu \mathrm{s}$, which is one OFDM symbol length in 3GPP LTE. Fig. 2 shows the average sum rate with respect to (w.r.t.) SNR during the updating period when $K=3, M=1$, and $L=100$. It is seen that the proposed algorithm and the SVD-based algorithm have nearly the same performance. However, the performance of the scheme that updates the beam only once every $L$ symbols and uses the same beam over the $L$ symbol period degrades considerably as the mobile speed increases. Figs. 3 and 4 show the sum rate loss by the proposed algorithm as a function of $p$ and as a function of $v_{m}$, respectively. Here, we use $c^{\prime}=0.102$ which is numerically obtained. The sum rate loss increases as the update instance becomes far from the reference beam instance, and it also increases as the mobile velocity increases, as expected. It is seen that the predicted behavior of the rate loss in Theorem 2 is consistent with the simulation result. Note that the sum rate loss by the proposed beam tracking method compared with the full subspace decomposition method is almost negligible.

\section{Conclusions}

We have proposed an efficient algorithm for tracking the beamforming matrices for the ZF CB in slowly-fading multicell MIMO channels, based on the null space perturbation theory, and have analyzed the sum rate loss of the proposed

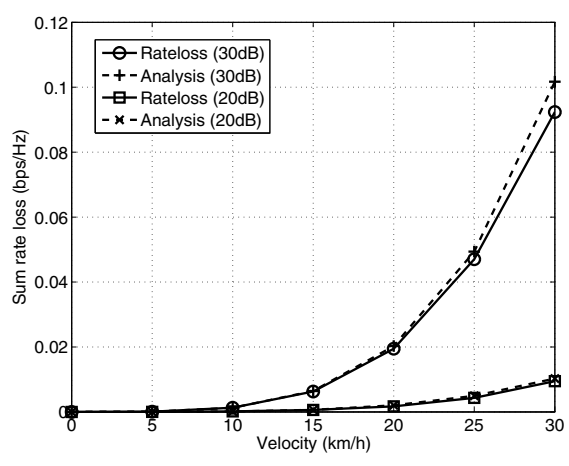

Fig. 4. Sum rate loss w.r.t. $v_{m}$ when $K=3, M=1$ and $p=10$.

algorithm. The proposed algorithm provides almost the same performance as the full subspace decomposition method with significantly reduced computational complexity. Thus, the proposed algorithm provides a very efficient transmit beam design method for multi-cell MIMO systems when the ZF criterion is used for coordinated beam design. The proposed algorithm can be applied to OFDM systems in the slowlyvarying case that beamforming matrices need to be updated during the transmission period. The simplest way is to apply the proposed algorithm to each subcarrier independently, or one can group OFDM subcarriers into several subcarrier blocks based on the channel coherence bandwidth, and can apply the proposed algorithm to each subcarrier block. In this way, the beamforming matrices can be effectively tracked.

\section{REFERENCES}

[1] 3GPP TR 36.814, V9.0.0, "Further advancements for EUTRA physical layer aspects," Mar. 2010.

[2] M. Sawahashi, Y. Kishiyama, A. Morimoto, D. Nishikawa and M. Tanno, "Coordinated multipoint transmission/reception techniques for LTE-Advanced," IEEE Wireless Commun., vol. 17, no. 3, pp. 26-34, June 2010.

[3] M. K. Karakayali, G. J. Foschini, and R. A. Valenzuela, "Network coordination for spectrally efficient communications in cellular systems," IEEE Wireless Commun., vol. 13, no. 4, pp. 56-61, Aug. 2006.

[4] J. Zhang, R. Chen, J. G. Andrews, A. Ghosh, and R. W. Heath, Jr., "Networked MIMO with clustered linear precoding," IEEE Trans. Wireless Commun., vol. 8, no. 4, pp. 1910-1921, Apr. 2009.

[5] L. Venturino, N. Prasad, and X. Wang, "Coordinated linear beamforming in downlink multi-cell wireless networks," IEEE Trans. Wireless Commun., vol. 9, no. 4, pp. 1451-1461, Apr. 2010.

[6] K. E. Avrachenkov and M. Haviv, "Perturbation of null spaces with application to the eigenvalue problem and generalized inverses," Linear Algebra Appl., vol. 369, pp. 1-25, Aug. 2003.

[7] H. Yu, Y. Sung, H. Kim, and Y. H. Lee, "Beam tracking for interference alignment in slowly-fading MIMO interference channels: a perturbations approach under a linear framework," IEEE Trans. Signal Process., vol. 60, no. 4, pp. 1910-1926, Apr. 2012.

[8] Q. H. Spencer, A. L. Swindlehurst, and M. Haardt, "Zero-forcing methods for downlink spatial multiplexing in multiuser MIMO channels," IEEE Trans. Signal Process., vol. 52, no. 2, pp. 461-471, Feb. 2004.

[9] G. H. Golub and C. F. V. Loan, Matix Computations, 2nd edition. The Johns Hopkins University Press, 1989.

[10] N. Jindal, "MIMO broadcast channels with finite-rate feedback," IEEE Trans. Inf. Theory, vol. 52, no. 11, pp. 5045-5060, Nov. 2006.

[11] M. Dong, L. Tong, and B. M. Sadler, "Optimal insertion of pilot symbols for transmissions over time-varying flat fading channels," IEEE Trans. Signal Process., vol. 52, no. 5, pp. 1403-1418, May 2004.

[12] C. Komninakis, C. Fragouli, A. H. Sayed, and R. D. Wesel, "Multiinput multi-output fading channel tracking and equalization using Kalman estimation," IEEE Trans. Signal Process., vol. 50, no. 5, pp. 1065-1076, May 2002. 